\title{
Verapamil versus hydrochlorothiazide in the treatment of hypertension: results of long term double blind comparative trial
}

\author{
Heinrich Holzgreve, Armin Distler, Joerg Michaelis, Thomas Philipp, Stefan Wellek, on behalf of \\ the Verapamil versus Diuretic (VERDI) Trial Research Group
}

\begin{abstract}
Objective-To compare the efficacy and tolerability of hydrochlorothiazide, sustained release verapamil, and their combination in patients with mild to moderate hypertension.

Design-Randomised multicentre trial of 48 weeks' duration with a double blind comparison of hydrochlorothiazide and verapamil followed by an open trial of combined treatment for patients not achieving the target diastolic blood pressure $(<90 \mathrm{~mm} \mathrm{Hg}$ ) during treatment with a single drug.

Setting-Outpatient departments in 10 clinics and 10 private practices of general practitioners or internists.

Patients - 369 Hypertensive patients with a diastolic blood pressure of $95-120 \mathrm{~mm} \mathrm{Hg}$ during a placebo run in period of two weeks.

Interventions-Initial treatment consisted of $12.5 \mathrm{mg}$ hydrochlorothiazide $(\mathrm{n}=187)$ or $120 \mathrm{mg}$ sustained release verapamil $(n=182)$ once daily (regimen I). If the target diastolic blood pressure of $<90 \mathrm{~mm} \mathrm{Hg}$ was not achieved within four weeks doses were increased to $25 \mathrm{mg}$ hydrochlorothiazide or $240 \mathrm{mg}$ verapamil once (regimen II) and twice daily (regimen III). Patients not achieving target blood pressure were given the combination of hydrochlorothiazide and verapamil-that is, 25 and $240 \mathrm{mg}$ once (regimen IV) and twice daily (regimen V).
\end{abstract}

Main outcome measure-Blood pressure determined with a device permitting automatic repeated measurements with printouts.

Results-After eight weeks of treatment with a single drug 76 out of $178(43 \%)$ and 101 out of 175 $(58 \%)$ patients achieved the target blood pressure with hydrochlorothiazide and verapamil, respectively. During follow up until 48 weeks patients treated with verapamil reached the target blood pressure more often and at lower doses and were less likely to switch to combination treatment than patients randomised to hydrochlorothiazide treatment. Adding verapamil to hydrochlorothiazide was more effective than the addition of hydrochlorothiazide to verapamil. At the end of the study 42 out of $169(25 \%)$ and 73 out of $163(45 \%)$ patients initially randomised to hydrochlorothiazide and verapamil, respectively, were at target blood pressure without combination treatment. After adding verapamil to hydrochlorothiazide or hydrochlorothiazide to verapamil an additional $58(34 \%)$ and $29(18 \%)$ patients reached the target blood pressure, respectively. Altogether 92 out of $332(28 \%)$ patients failed to achieve target blood pressure with regimen $\mathrm{V}$. There were four, 10, seven, and seven withdrawals due to possible adverse effects to treatment with hydrochlorothiazide, verapamil, combining verapamil with hydrochlorothiazide, and combining hydrochlorothiazide with verapamil, respectively.

Conclusions-In doses currently used in antihypertensive treatment verapamil was more effective than hydrochlorothiazide as a single agent and in combination in mild to moderate hypertension, whereas withdrawal rates caused by side effects possibly related to treatment were similar.

\section{Introduction}

Over the past decade several new drugs have been introduced to treat hypertension, thus providing alternative options for treatment. Many authorities and expert organisations world wide, however, still recommend thiazide diuretics and $\beta$ blockers as first line drugs for the treatment of hypertension. ${ }^{12}$ As single agents or with the addition of other agents, the two kinds of drugs have been compared as initial treatment in three long term controlled trials and could not be shown to affect differentially overall cardiovascular morbidity and mortality. ${ }^{3.5}$ There has been concern, however, about the potential disadvantages of both these groups of antihypertensive agents, such as subjective side effects and electrolyte or metabolic disturbances.

In the past few years calcium antagonists have been recommended for the treatment of hypertension. ${ }^{67}$ The selection of a new antihypertensive agent or a modification of the commonly used stepped care approach to antihypertensive treatment, however, presupposes that the new drug has been proved to be superior, or at least equivalent to, the standard treatment. Thus there is a need for long term studies of sufficient numbers of patients to compare the antihypertensive efficacy, tolerability, and adverse drug reactions of the new therapeutic regimen with the standard treatment. Therefore, in a double blind controlled study over one year we evaluated the thiazide diuretic hydrochlorothiazide and the calcium antagonist verapamil within the scope of a comparative therapeutic trial in patients with mild to moderate hypertension.

\section{Patients and methods}

The design was a multicentre, randomised, double blind, controlled clinical trial with a single blind placebo baseline period of two weeks. This was preceded by an additional wash out period of two weeks for patients who had been previously treated with antihypertensive drugs. The study population comprised male and female outpatients who had $(n=239)$ or had not been treated $(n=130)$; their age range was $22-71$ and they had a resting diastolic blood pressure of $95-120 \mathrm{~mm} \mathrm{Hg}$ while sitting. Exclusion criteria were a diastolic pressure $<95$ or $>120 \mathrm{~mm} \mathrm{Hg}$ at the start or at the end of the two week placebo run in
Correspondence to:

Professor Holzgreve.

BrMed f 1989;299:881-6 
period; frank congestive heart failure; myocardial infarction within the past six months; secondary and tertiary atrioventricular block; sick sinus syndrome; sinus bradycardia ( $<50$ beats/min); treatment with diuretics or calcium antagonists for other diseases; hyperuricaemia or hyperlipidaemia requiring treatment with drugs; a history or evidence of malignancy or other serious diseases sufficient to interfere with long term adherence to the trial protocol; $\geqslant 30 \%$ overweight (Broca index); pregnancy; the use of oral contraceptives; serum creatinine concentration $>200 \mu \mathrm{mol} / \mathrm{l}$; and hypokalaemia $<3.6 \mathrm{mmol} / \mathrm{l}$. Patients were included only on giving consent after full explanation of the nature and purpose of the investigation. The study protocol was approved by the ethics committee of the German League against Hypertension.

To ensure standardised blood pressure recordings in all centres of the trial blood pressure was measured by means of an automatic device (Tonoprint electronic, Speidel and Keller, 7455 Jungingen, Federal Republic of Germany). This device records the Korotkoff sounds by microphone and the diastolic blood pressure is registered at phase $\mathrm{V}$. The blood pressure values are printed with the date and time of the day. An identical version of the measuring device without a printout (Tonomed) has been tested. ${ }^{8}$ The Tonoprint was validated by direct comparison with the conventional sphygmomanometer in a separate group of 65 normotensive and hypertensive patients. Twelve recordings were taken with each method alternately in each patient (H Holzgreve et al, unpublished results). Diastolic pressure was comparable by the two techniques $(83.80$ (SD 13.86) $\mathrm{mm} \mathrm{Hg}$ with the Tonoprint and $84.50(13.64) \mathrm{mm} \mathrm{Hg}$ with the sphygmomanometer. The reproducibility of diastolic values was also similar with the two methods: averaging the 65 within series standard deviations gave $4.35(2 \cdot 76) \mathrm{mm} \mathrm{Hg}$ for Tonoprint and $3.50(1.44) \mathrm{mm} \mathrm{Hg}$ for the sphygmomanometer.

At each visit blood pressure and heart rate were measured as closely as possible to 24 hours after the last treatment during the placebo period and regimens $I$, II, and IV (once daily treatment) or to 12 hours during regimens III and V (twice daily treatment). Six recordings were taken while the patient was sitting every two minutes after five minutes' rest. All decisions about admission to the study and changes in treatment were based on the average of the first five blood pressure recordings. To minimise inclusion of technical errors (which may occur if the cuff containing the microphone is not placed correctly) single blood pressure recordings that differed by more than $15 \mathrm{~mm} \mathrm{Hg}$ from the mean of the first five measurements were discarded and the value of the sixth measurement was included in the calculation of the mean value instead. If two measurements had to be discarded the entire measurement procedure was repeated. The printouts with date, time, blood pressure, and heart rate were included in the follow up records and checked by the central office.

Between July 1985 and December 1986 a total of 376 patients were admitted to the trial at 20 centres, 10 each at clinical outpatient departments and in private practices of general practitioners or internists. Two centres with seven patients were excluded during the early phase of the trial: one after inclusion of six patients because the automatic device for blood pressure recording was not used and the other because the doctor in charge took over a new position after one patient had been enrolled. Of the remaining 369 patients, 187 were randomised to hydrochlorothiazide treatment and 182 to verapamil.

The drugs were specially manufactured for study purposes and tested for appropriate bioavailability.
They consisted of identical oblong tablets with a dividing groove containing either $25 \mathrm{mg}$ hydrochlorothiazide or $240 \mathrm{mg}$ sustained release verapamil (Isoptin SR, Knoll AG, Ludwigshafen, Federal Republic of Germany). Treatment for six patients with the study number were sent to each centre in advance to be given to the patients entering the study according to the order of the randomised numbers.

Treatment was started with either $12.5 \mathrm{mg}$ hydrochlorothiazide or $120 \mathrm{mg}$ verapamil (regimen I). Mandatory follow up visits were after two, four, six, eight, 12, 24, 36, and 48 weeks. Additional visits were scheduled two weeks after the start of a new regimen. If the target blood pressure, defined as a diastolic pressure $<90 \mathrm{~mm} \mathrm{Hg}$, was not achieved after two weeks (as in regimen I after four weeks) the next regimen was prescribed. These were $25 \mathrm{mg}$ hydrochlorothiazide or $240 \mathrm{mg}$ verapamil once daily (regimen II) or twice daily (regimen III). Patients who did not reach the target blood pressure on randomised regimen III at eight weeks or at a later follow up visit were treated in an open study with a combination of hydrochlorothiazide and verapamil - that is, 25 and $240 \mathrm{mg}$ once daily (regimen IV) or twice daily (regimen V). Patients remained taking active treatment for 48 weeks. If at one visit during the study target blood pressure was not achieved after at least two weeks at the highest dose (regimen V) patients were withdrawn from the study for reasons related to treatment. Other reasons for discontinuation of treatment were specified as: in study diastolic blood pressure $>120 \mathrm{~mm} \mathrm{Hg}$; severe adverse effects; non-compliance (for example, interruption or change of randomised treatment by doctors not from the study or by the patient or by failure of the patient to meet appointments); development of a new serious disease unrelated to treatment; emergence of a new exclusion criterion; and other causes.

At entry and after eight and 48 weeks of active treatment blood samples for laboratory evaluations and an electrocardiogram were taken and a structured questionnaire concerning possible side effects, tolerability, and general wellbeing were filled out by a doctor or nurse. The biochemical profile included measuring blood glucose concentrations and concentrations of potassium, creatinine, urate, glycated haemoglobin, total, low density lipoprotein, and high density lipoprotein cholesterol, and triglycerides. All these analyses were made centrally in specialised laboratories. At each centre the doctors in charge were individually responsible for all examinations and basic laboratory tests necessary for the immediate care of the patients. Supplementation with potassium chloride or the use of potassium sparing diuretics was permitted in patients whose serum potassium concentrations became $<3.6 \mathrm{mmol} / \mathrm{l}$.

The rates of response for the two treatments were tested for homogeneity by means of Fisher's exact test for $2 \times 2$ contingency tables. In this step of the analysis significance was set at $5 \%$. Numerous additional tests were performed in an explorative rather than confirmative manner. In particular, several sets of $2 \times 2$ contingency subtables produced by stratification with respect to some categorical covariables were tested for homogeneity of the odds ratio. For this purpose the statistic proposed by Breslow and Day was used."

\section{Results}

\section{COMPARABILITY OF GROUPS}

The 369 patients who entered the trial were randomised to hydrochlorothiazide $(n=187)$ and verapamil $(n=182)$. On entry into the double blind part of the study the two groups were well balanced with regard to systolic and diastolic blood pressures, heart rate, sex, 
weight, and previous treatment (table I). Patients randomised to verapamil treatment were 3.3 years older than those given hydrochlorothiazide.

\section{WITHDRAWALS}

During the study 37 patients ( 15 given hydrochlorothiazide, 10 given verapamil, and 12 given both) withdrew for reasons considered to be unrelated to treatment, mainly because of patient non-compliance or protocol violation by family doctors not participating in the study, inability to keep the regular appointments, new serious diseases, and emergence of a new exclusion criterion (table II).

In addition, 38 patients discontinued active treatment because of adverse effects $(n=28)$ or an increase of diastolic blood pressure above $120 \mathrm{~mm} \mathrm{Hg}(\mathrm{n}=10)$. Furthermore, 79 patients were withdrawn from the study because they did not achieve the target blood pressure of $<90 \mathrm{~mm} \mathrm{Hg}$ diastolic after being at least two weeks on the highest combination dose (regimen V).

\section{RATES OF RESPONSE}

The cumulative numbers of withdrawals unrelated to treatment until weeks 8,24 , and 48 were 16,28 , and 37 , respectively. The remaining 353,341 , and 332 patients yielded data for evaluation of the efficacy of the drugs at eight, 24, and 48 weeks of active treatment (table III). Within the first eight weeks there was a significant difference in the number of patients who achieved the target blood pressure during treatment with one drug (76 out of $178.43 \%$ ) taking hydrochlorothiazide $v 101$ out of $175(58 \%)$ taking verapamil; $\mathrm{p}=0.0048)$. The $95 \%$ confidence interval for the observed $15 \%$ treatment difference was $4 \cdot 7 \%$ to $25 \%$.

TABLE I - Characteristics of patients at entry to study. Values are means (SD) unless stated otherwise

\begin{tabular}{lcc}
\hline & $\begin{array}{c}\text { Hydrochlorothiazide } \\
(\mathbf{n}=187)\end{array}$ & $\begin{array}{c}\text { Verapamil } \\
(\mathbf{n}=182)\end{array}$ \\
\hline Blood pressure $(\mathrm{mm} \mathrm{Hg}):$ & & \\
$\quad$ Systolic & $152 \cdot 6(16 \cdot 2)$ & $152 \cdot 3(15 \cdot 3)$ \\
$\quad$ Diastolic & $104 \cdot 4(6 \cdot 4)$ & $104 \cdot 2(6 \cdot 1)$ \\
Heart rate $($ beats/min) & $78 \cdot 0(12 \cdot 2)$ & $79 \cdot 4(12 \cdot 8)$ \\
Age $(y e a r s)$ & $48 \cdot 8(10 \cdot 7)$ & $52 \cdot 1(10 \cdot 7)$ \\
Sex, $\mathrm{M} / \mathrm{F}(\%)$ & $49 \cdot 7 / 50 \cdot 3$ & $58 \cdot 0 / 42 \cdot 0$ \\
Weight $(\mathrm{kg})$ & $75 \cdot 2(11 \cdot 6)$ & $76 \cdot 9(11 \cdot 4)$ \\
Height $(\mathrm{cm})$ & $170 \cdot 3(7 \cdot 7)$ & $171 \cdot 4(8 \cdot 0)$ \\
Previous antihypertensive treatment $(\%(\mathrm{No})):$ & $38(71)$ & $32(59)$ \\
$\quad$ None & & $15(26)$ \\
Treated with & $15(27)$ & $32(58)$ \\
$\quad$ Diuretic & $35(64)$ & $21(37)$ \\
$\quad$ Blocker & $14(25)$ & $27(48)$ \\
$\quad$ Ther antihypertensive agents & $26(48)$ & \\
\hline
\end{tabular}

TABLE III - Numbers (percentages) of patients achieving and not achieving target blood pressure with different regimens of treatment and numbers (percentages) withdrawn for reasons related to treatment

\begin{tabular}{|c|c|c|}
\hline & \multicolumn{2}{|c|}{ Randomised to: } \\
\hline & Hydrochlorothiazide & Verapamil \\
\hline & After 8 weeks & \\
\hline \multirow{2}{*}{\multicolumn{3}{|c|}{$\begin{array}{l}\text { Target pressure: } \\
\text { Achieved }\left(\text { regimen }^{\star}\right):\end{array}$}} \\
\hline & & \\
\hline & $6(3)$ & $14(8)$ \\
\hline II & $29(16)$ & $36(21)$ \\
\hline III & $41(23)$ & $51(29)$ \\
\hline Not achieved & $94(53)$ & $65(37)$ \\
\hline Withdrawn & $8(5)$ & $9(5)$ \\
\hline \multirow[t]{2}{*}{ Total } & 178 & 175 \\
\hline & After 24 weeks & \\
\hline \multicolumn{3}{|c|}{ Target pressure: } \\
\hline \multicolumn{3}{|c|}{ Achieved $\left(\right.$ regimen $\left.{ }^{\star}\right)$ : } \\
\hline I & $2(1)$ & $10(6)$ \\
\hline II & $20(12)$ & $39(23)$ \\
\hline III & $24(14)$ & $30(18)$ \\
\hline IV & $44(26)$ & $21(12)$ \\
\hline V & $37(22)$ & $23(14)$ \\
\hline Not achieved & $33(19)$ & $26(15)$ \\
\hline Withdrawn & $12(7)$ & $20(12)$ \\
\hline \multirow[t]{2}{*}{ Total } & 172 & 169 \\
\hline & After 48 weeks & \\
\hline \multirow{2}{*}{\multicolumn{3}{|c|}{ Target pressure: }} \\
\hline & \multicolumn{2}{|c|}{ Achieved $\left(\right.$ regimen $\left.{ }^{\star}\right)$ : } \\
\hline I & $2(1)$ & $5(3)$ \\
\hline II & $21(12)$ & $35(22)$ \\
\hline III & $19(11)$ & $33(20)$ \\
\hline IV & $24(14)$ & $15(9)$ \\
\hline V & $34(20)$ & $14(9)$ \\
\hline Not achieved & $54(32)$ & $38(23)$ \\
\hline Withdrawn & $15(9)$ & $23(14)$ \\
\hline Total & 169 & 163 \\
\hline
\end{tabular}

*Regimens: $\mathrm{I}=12.5 \mathrm{mg}$ hydrochlorothiazide or $120 \mathrm{mg}$ verapamil once daily; $\mathrm{II}=25 \mathrm{mg}$ hydrochlorothiazide or $240 \mathrm{mg}$ verapamil once daily; III $=25 \mathrm{mg}$ hydrochlorothiazide or $240 \mathrm{mg}$ verapamil twice daily; IV $=25 \mathrm{mg}$ hydrochlorothiazide and $240 \mathrm{mg}$ verapamil once daily: $\mathrm{V}=25 \mathrm{mg}$ hydrochlorothiazide and $240 \mathrm{mg}$ verapamil twice daily.

With all the regimens target blood pressure was achieved more often with verapamil. The cumulative rates of response for verapamil were $8 \%(14 / 175)$ with regimen I, 29\% (50/175) with regimens I and II, and $58 \%(101 / 175)$ with regimens I, II, and III compared with $3 \%(6 / 178), 20 \%(35 / 178)$, and $43 \%(76 / 178)$ for hydrochlorothiazide, respectively (table III).

After 24 and 48 weeks of active treatment the results were similar (table III). More patients taking verapamil $(47 \%(79 / 169)$ and $45 \%(73 / 163))$ than hydrochlorothiazide $(27 \%(46 / 172)$ and $25 \%(42 / 169))$ achieved a diastolic blood pressure below $90 \mathrm{~mm} \mathrm{Hg}$ $(p=0.0001 ; 95 \%$ confidence interval for the observed treatment difference $10 \%$ to $30 \%$ ). With all regimens

TABLE II - Reasons for withdrawal from active treatment. Values are numbers of patients

\begin{tabular}{|c|c|c|c|c|c|}
\hline & \multirow[b]{2}{*}{$\begin{array}{c}\text { Hydrochlorothiazide } \\
\text { alone } \\
(n=67)\end{array}$} & \multirow[b]{2}{*}{$\begin{array}{l}\text { Verapamil } \\
\text { alone } \\
(\mathrm{n}=100)\end{array}$} & \multicolumn{2}{|c|}{ Combination treatment } & \multirow[b]{2}{*}{$\begin{array}{c}\text { Total } \\
\langle\mathbf{n}=369)\end{array}$} \\
\hline & & & $\begin{array}{c}\text { Adding } \\
\text { verapamil to } \\
\text { hydrochlorothiazide } \\
(\mathrm{n}=120)\end{array}$ & $\begin{array}{l}\text { Adding } \\
\text { hydrochlorothiazide } \\
\text { to verapamil } \\
(\mathbf{n}=82)\end{array}$ & \\
\hline Administrative causes & 15 & 10 & 3 & 9 & 37 \\
\hline Patient non-compliance & 9 & 7 & 3 & 4 & 23 \\
\hline Protocol violation by non-study doctor & 3 & & & 2 & 5 \\
\hline New disease & & $2^{\star}$ & & $2+$ & 4 \\
\hline New exclusion criterion & & 1 & & 1 & 2 \\
\hline Change of residence & 2 & & & & 2 \\
\hline Voluntary weight loss $17 \mathrm{~kg}$ ) & 1 & & & & 1 \\
\hline Non-administrative causes & 8 & 15 & 7 & 8 & 38 \\
\hline Adverse effects & $4 \ddagger$ & 105 & 7 & 79 & 28 \\
\hline Diastolic pressure $>120 \mathrm{~mm} \mathrm{Hg}$ & 4 & 5 & & 1 & 10 \\
\hline Target blood pressure not achieved & & & 44 & 35 & 79 \\
\hline Total & 23 & 25 & 54 & 52 & 154 \\
\hline
\end{tabular}

$\star$ Breast and pancreatic cancer.

†Gynaccological tumour and lumbar disc prolapse, each requiring operation.

fNausea (2), impotence, and orthostatic hypotension.

Rash (2), short lived dysphasia, drug induced cholestatic hepatitis, orthostatic hypotension, dizziness, palpitation, impotence, abdominal cramps, and

urinary retention.
|Constipation (4), oedema, impotence, and weakness.

Oedema (3), constipation, dizziness, gastric discomfort, and weakness. 
verapamil was effective in more patients than hydrochlorothiazide. The cumulative rates of response for verapamil and hydrochlorothiazide after 24 weeks were respectively $6 \%$ and $1 \%$ (regimen I), $29 \%$ and $13 \%$ (regimens I and II), and $47 \%$ and $27 \%$ (regimens I, II, and III). The corresponding rates after 48 weeks were $3 \%$ and $1 \%, 25 \%$ and $14 \%$, and $45 \%$ and $25 \%$.

According to subgroup analyses, similar results were obtained in male and female patients as well as in patients with and without previous antihypertensive treatment and in patients from clinical outpatient departments and private practices. The target diastolic blood pressure was achieved more often with verapamil than with hydrochlorothiazide. With both drugs the rates of response increased with age. At all ages, however, treatment with verapamil alone reduced diastolic blood pressure more often below $90 \mathrm{~mm} \mathrm{Hg}$ than that with hydrochlorothiazide (table IV). A similar observation proved true for subgroups with lower and higher than median diastolic blood pressure at entry $(103 \mathrm{~mm} \mathrm{Hg})$ : in both groups patients achieved target blood pressure more often during treatment with verapamil alone than with hydrochlorothiazide alone. After both hydrochlorothiazide and verapamil, however, patients with lower initial blood pressure had higher rates of response than those with higher initial blood pressure (table IV).

If diastolic blood pressure was not reduced below $90 \mathrm{~mm} \mathrm{Hg}$ with treatment with a single drug, combination treatment was started. More patients randomised to hydrochlorothiazide achieved the target blood pressure by adding verapamil than patients randomised to verapamil who had hydrochlorothiazide added (at 24 weeks 81 out of $172(47 \%)$ and 44 out of $169(26 \%)$; at 48 weeks 58 out of $169(34 \%)$ and 29 out of $163(18 \%)$, respectively (table III). Therefore the total percentage of patients who reached the target blood pressure having taken a single drug and the two drugs combined was similar irrespective of the original randomised treatment - that is, 127 out of $172(74 \%)$ and 123 out of $169(73 \%)$ at 24 weeks and 100 out of $169(59 \%)$ and 102 out of $163(63 \%)$ at 48 weeks, respectively.
BLOOD PRESSURE

At entry the mean blood pressure was $152 \cdot 6(16 \cdot 2) /$ $104 \cdot 4(6 \cdot 4)$ and $152 \cdot 3(15 \cdot 3) / 104 \cdot 2(6 \cdot 1) \mathrm{mm} \mathrm{Hg}$ in the hydrochlorothiazide and verapamil group, respectively (table I). Increasing dose regimens were given to achieve the target blood pressure, and patients who did not reach the target having received the highest dose were withdrawn from the study. Therefore blood pressure is given for all patients remaining in the study rather than for each treatment group, which would be misleading. Altogether, blood pressure was reduced from $152 \cdot 4(15 \cdot 7) / 104 \cdot 3(6 \cdot 2)$ to $132 \cdot 1(15 \cdot 4) / 86 \cdot 4$ $(7 \cdot 1) \mathrm{mm} \mathrm{Hg}$ (table V). At all time points during the study, however, more patients remained taking verapamil alone than hydrochlorothiazide alone.

\section{ADVERSE EFFECTS}

The number of withdrawals because of major adverse effects and the complaints leading to withdrawal are listed in table II. Of these patients, four were in the hydrochlorothiazide group and 10 in the verapamil group. A further 14 patients taking combination treatment withdrew, seven adding verapamil to hydrochlorothiazide and seven after adding hydrochlorothiazide to verapamil.

During the study no unexpected adverse effects were observed. According to the structured questionnaire, patients complained of frequently having to urinate after hydrochlorothiazide and of constipation after verapamil. Potassium salts or potassium sparing diuretics were taken during the study by 17 and six patients treated with hydrochlorothiazide or verapamil, respectively. With the exception of a mean decrease of serum potassium concentration of $0.311 \mathrm{mmol} / 1$ $(p=0.0002)$ there was no substantial change in other laboratory values.

\section{Discussion}

This large, randomised, double blind, long term study was undertaken to compare the antihypertensive efficacy and the tolerability of the calcium antagonist

TABLE IV-Proportions ${ }^{\star}$ (percentages) of patients achieving target blood pressure after eight, 24, and 48 weeks of treatment with hydrochlorothiazide or verapamil

\begin{tabular}{|c|c|c|c|c|c|c|c|c|c|}
\hline & \multicolumn{3}{|c|}{ After 8 weeks } & \multicolumn{3}{|c|}{ After 24 weeks } & \multicolumn{3}{|c|}{ After 48 weeks } \\
\hline & Hydrochlorothiazide & $\mathrm{p}$ Value & Verapamil & Hydrochlorothiazide & $\mathrm{p}$ Value & Verapamil & Hydrochlorothiazide & $\mathrm{p}$ Value & Verapamil \\
\hline All patients & $76 / 178(+3)$ & $0 \cdot 0048$ & $101 / 175$ & $46 / 172(27)$ & 0.0001 & $79 / 169(47)$ & $42 / 169(25)$ & 0.0001 & $73 / 163(45)$ \\
\hline $\begin{array}{c}\text { Age years } \\
<+5 \\
45-55\end{array}$ & $\begin{array}{l}19 / 55(35 \\
31 / 68+6)\end{array}$ & \multirow{2}{*}{$\begin{array}{l}0.159 \dagger \\
0.3520 \dagger \\
|0.0767 \dagger| \\
|0.882 \ddagger|\end{array}$} & $\begin{array}{l}22 / 45(49) \\
28 / 50(56)\end{array}$ & $\begin{array}{l}11 / 53(21) \\
17 / 66(26)\end{array}$ & \multirow{2}{*}{$\begin{array}{l}0 \cdot 0436 \dagger \\
0 \cdot 1070 \dagger \\
\{0 \cdot 0329 \dagger \mid \\
0 \cdot 899 \ddagger\end{array}$} & $\begin{array}{l}17 / 42(41) \\
19 / 47(40)\end{array}$ & $\begin{array}{l}10 / 52(19) \\
14 / 65(22)\end{array}$ & \multirow{2}{*}{$\begin{array}{l}0.0981 \dagger \\
0.0334 \dagger \\
\left\{\begin{array}{l}0 \cdot 1030 \dagger \\
0.844 \ddagger\end{array}\right\}\end{array}$} & $\begin{array}{l}15 / 41(37) \\
19 / 45(42)\end{array}$ \\
\hline$\geqslant 55$ & $26 / 55(47)$ & & $51 / 80(64)$ & $18 / 53(34)$ & & $43 / 80(54)$ & $18 / 52(35)$ & & $39 / 77(51)$ \\
\hline \multicolumn{10}{|c|}{$\begin{array}{l}\text { Diastolic pressure at entry } \\
(\mathrm{mm} \mathrm{Hg}):\end{array}$} \\
\hline$\leqslant 103$ & $47 / 86(55)$ & \multirow{2}{*}{$\begin{array}{c}0.0297 \dagger \\
|0 \cdot 0904 \dagger| \\
0.726 \ddagger\end{array}$} & $63 / 89(71)$ & $27 / 81(33)$ & \multirow{2}{*}{$\left.\begin{array}{l}0 \cdot 0003 \dagger \\
\{0 \cdot 1230 \dagger \\
0 \cdot 193 \ddagger\end{array}\right\}$} & $53 / 86(62)$ & $23 / 79(29)$ & $0.0003 \uparrow$ & $49 / 84(58)$ \\
\hline$>103$ & $29 / 92(32)$ & & $38 / 86(44)$ & $19 / 91(21)$ & & $26 / 83(31)$ & $19 / 90(21)$ & $\left\{\begin{array}{l}0 \cdot 2150 \dagger \\
0 \cdot 129 \ddagger\end{array}\right\}$ & $24 / 79(30)$ \\
\hline
\end{tabular}

*Number of patients at target blood pressure divided by total number of patients in subgroup excluding patients withdrawn for administrative causes.

†For comparison of hydrochlorothiazide with verapamil in the subgroups by means of Fisher's exact test.

$\ddagger$ Associated with Breslow-Day statistic for homogeneity of several odds ratios testing whether the superiority of verapamil relative to hydrochlorothiazide depends on age or diastolic pressure at entry.

TABLE V-Mean (SD) blood pressures and numbers of patients receiving each drug regimen during weeks of treatment

\begin{tabular}{|c|c|c|c|c|c|c|}
\hline & \multicolumn{6}{|c|}{ Week of treatment } \\
\hline & 0 & 8 & 12 & 24 & 36 & 48 \\
\hline \multicolumn{7}{|l|}{ Blood pressure $(\mathrm{mm} \mathrm{Hg})$ : } \\
\hline Systolic & $152.4(15 \cdot 7)$ & $138 \cdot 7(15 \cdot 1)$ & $137 \cdot 3(15 \cdot 0)$ & $133 \cdot 7(14 \cdot 1)$ & $132 \cdot 5(14 \cdot 2)$ & $132 \cdot 1(15 \cdot 4)$ \\
\hline Diastolic & $104 \cdot 3(6 \cdot 2)$ & $93 \cdot 4(9 \cdot 2)$ & $91 \cdot 3(9 \cdot 1)$ & $88 \cdot 2(7 \cdot 6)$ & $87 \cdot 1(7 \cdot 1)$ & $86 \cdot 4(7 \cdot 1)$ \\
\hline \multicolumn{7}{|l|}{ Patients receiving: } \\
\hline Hydrochlorothiazide alone & 187 & 170 & 92 & 60 & 48 & 44 \\
\hline Verapamil alone & 182 & 166 & 106 & 86 & 77 & 75 \\
\hline Hydrochlorothiazide and & & & 74 & 85 & 71 & 66 \\
\hline \multicolumn{7}{|l|}{ Verapamil and } \\
\hline hydrochlorothiazide & & & 54 & 54 & 41 & 30 \\
\hline Total & 369 & 336 & 326 & 285 & 237 & 215 \\
\hline
\end{tabular}

verapamil in its sustained release form with a standard drug for antihypertensive treatment, the thiazide diuretic hydrochlorothiazide. The randomisation resulted in good comparability between the two treatment groups. The observed minor difference in the mean age associated with a small $p$ value based on Student's $t$ test is too small to explain any major difference in rates of response. In fact, the results of a logistic regression analysis suggested that increasing age from $48 \cdot 8$ to $52 \cdot 1$ years (table I) accounted for a difference of $2 \cdot 6 \%$ between response rates for the first eight weeks.

In evaluating the results three major methodological aspects must be considered. Firstly, the primary goal of treatment was a diastolic blood pressure of less than 
$90 \mathrm{~mm} \mathrm{Hg}$ - that is, whenever the diastolic blood pressure was not reduced below $90 \mathrm{~mm} \mathrm{Hg}$ the next dose step of hydrochlorothiazide or verapamil was given and if necessary both drugs were combined. Patients not achieving the target blood pressure with the highest dose regimen $(\mathrm{V})$ were withdrawn from the study. Therefore the design of the protocol implies that the two drugs tested must be compared by the number and the rate of patients achieving the target blood pressure during the double blind period of the study rather than by the reduction in blood pressure. Secondly, the combination of the two drugs in the open part of the study answers the question whether the efficacy of the combination is dependent on the type of drug used to start treatment. Thirdly, in contrast with many other multicentre studies the present trial took advantage of an automatic blood pressure device with printouts ensuring standardisation and verification of the measurements.

In our patients with diastolic blood pressures of 95 to $120 \mathrm{~mm} \mathrm{Hg}$ at entry more patients achieved the target blood pressure with verapamil alone than with hydrochlorothiazide alone. Thus with verapamil 58\%, 47\%, and $45 \%$ of patients showed a reduction in diastolic pressure to $<90 \mathrm{~mm} \mathrm{Hg}$ after eight, 24, and 48 weeks of active treatment compared with $43 \%, 27 \%$, and $25 \%$ with hydrochlorothiazide. Verapamil proved to be significantly more effective than hydrochlorothiazide with all regimens. Therefore more patients remained taking verapamil alone $(n=75)$ than hydrochlorothiazide alone $(n=44)$ until the end of the study. With respect to the response rate-that is, percentage of patients achieving the target diastolic blood pressure of $<90 \mathrm{~mm} \mathrm{Hg}$-verapamil was superior to hydrochlorothiazide also in several subgroups defined afterwards by sex, pretreatment state, care in clinics or practices, and age. These results corroborate our main findings.

The percentage of responders in our study seems rather low. Higher rates of response have, however, been reported, mostly from uncontrolled trials. In our study an adequate placebo period preceded the start of drug treatment, the mean blood pressure at entry was rather high, and a low target blood pressure was chosen. Thus a patient was defined as a non-responder if he or she failed to achieve a diastolic blood pressure of $<90 \mathrm{~mm} \mathrm{Hg}$ at a single visit. When the doses used and the blood pressures at entry are taken into account the efficacy of hydrochlorothiazide in lowering blood pressure in our study compares adequately with results from other controlled trials. ${ }^{11 \cdot 12}$

Verapamil has been shown to be superior to hydrochlorothiazide in the three regimens used. It could be argued that this was because of our failure to select equipotent doses of the two drugs. Daily doses of hydrochlorothiazide between 12.5 and $25 \mathrm{mg}$ were, however, selected for the following reasons. Firstly, studies using doses of hydrochlorothiazide of up to $200 \mathrm{mg}$ daily have not shown convincing evidence of an increased blood pressure lowering effect with doses $\geqslant 50 \mathrm{mg}$ daily. 11113 Secondly, in recent years titration to the lowest possible dose of antihypertensive agents has been suggested to avoid possible subjective side effects and adverse metabolic alterations.' In a double blind cross over study a daily dose of $12.5 \mathrm{mg}$ hydrochlorothiazide gave almost the same reduction in blood pressure as doses of 25 and $50 \mathrm{mg}$ daily after six weeks of treatment. ${ }^{13}$ Therefore we chose a daily dose of $12.5 \mathrm{mg}$ as the lowest dose. Although doses between 12.5 and $50 \mathrm{mg}$ daily used in our study are commonly recommended, our intention to select the lowest possible dose range might have resulted in the administration of submaximally effective doses of hydrochlorothiazide. In fact, only two out of $172(1 \%)$ and two out of $169(1 \%)$ patients reached the target blood pressure with a daily dose of $12.5 \mathrm{mg}$ hydrochlorothiazide after 24 and 48 weeks of treatment, respectively. On the other hand, the same strategy led us to choose a daily dose of $120 \mathrm{mg}$ verapamil, for which an antihypertensive effect had not been shown at the time we started our study. According to our protocol, the blood pressure was measured at the end of the dosing interval and before the administration of the next dose. Therefore another possible, but rather unlikely, explanation for the higher response rates with verapamil compared with hydrochlorothiazide is that the duration of action of sustained release verapamil is longer than that of hydrochlorothiazide.

Combining the two drugs tested in patients who did not achieve the target blood pressure with a single drug resulted in higher rates of response when verapamil was added to hydrochlorothiazide than when hydrochlorothiazide was added to verapamil. Therefore the total response rate using single drug treatment and combining the two drugs was almost identical $(74 \%$ and $73 \%$ at 24 weeks and $59 \%$ and $63 \%$ at 48 weeks; table III) and independent of whether treatment was started with the diuretic or the calcium antagonist. On the other hand, this implies that a considerable proportion of patients will achieve the target blood pressure with verapamil alone who would need combination treatment if they were first treated with hydrochlorothiazide.

Each instance of a patient refusing to take the drug or to continue to attend the scheduled visits because of side effects was coded as a treatment related cause. The number of adverse effects leading to withdrawal from randomised treatment was low for both drugs. There were more withdrawals possibly related to adverse effects during treatment with verapamil alone than with hydrochlorothiazide alone ( $10 v$ four). Because of higher rates of response, however, more patients were taking verapamil alone for longer.

Discontinuation was due to intolerable adverse effects, some of which accorded well with previous experience of the two drugs, including the one case of drug induced cholestatic hepatitis, which has been reported in a few patients for verapamil, nifedipine, and diltiazem..$^{1+15}$ Others may be judged as non-specific symptoms of antihypertensive treatment. Remarkably, some complaints leading to withdrawal of patients by the blinded doctors had not been previously recognised as side effects of the drugs and might not be related to treatment at all. Furthermore, constipation or oedema, well known side effects of calcium antagonists, did not cause discontinuation in any patient during treatment with verapamil alone. Four patients were, however, withdrawn because of constipation and three patients because of oedema after combining verapamil with hydrochlorothiazide and hydrochlorothiazide with verapamil, respectively. These inconsistencies with present knowledge may be taken as evidence that studies with a higher number of patients are needed to determine the profile of severe adverse effects of drugs with low rates of such side effects. For these reasons we have refrained from calculating standardised withdrawal rates.

In conclusion, in this long term, randomised, double blind trial treatment with sustained release verapamil alone more often lowered diastolic blood pressure below $90 \mathrm{~mm} \mathrm{Hg}$ than hydrochlorothiazide in doses currently used in antihypertensive treatment. Furthermore, adding verapamil to hydrochlorothiazide was more effective in controlling high blood pressure than combining hydrochlorothiazide with verapamil. There were no major differences in the number of withdrawals for medical or administrative reasons. Therefore our results suggest that in doses currently used in mild to moderate hypertension the sustained release form of the calcium antagonist verapamil is more 
effective than hydrochlorothiazide in a substantial number of hypertensive patients.

The Verapamil versus Diuretic (VERDI) Trial Research Group comprises: Steering committee - A Distler, H Holzgreve (chairman), J Michaelis, T Philipp. Working party-A Distler (Berlin), A Eckardt (Ludwigshafen), W Gauß (Ludwigshafen), G Hilgenstock (Berlin), H Holzgreve (Munich), J Michaelis (Mainz), G Pfennigsdorf (Ludwigshafen), T Philipp (Essen), S Wellek (Mainz). Investigators J Kindler, B Heintz (Aachen), D Kraft, W Pusch, H Reitzig (Berlin), B Schmaltz (Bingen), R M Lederle (Dortmund), W Kirch, G Wensing, G Loose (Essen and Kiel), E Keidl (Geisenheim), H C Burck (Kiel), H W Karch (Kirn), C Osiecki (Koblenz), B Hinkelmann (Kuppenhein), W D Pfund (Lauf an der Pegnitz), K H Rahn, R Böhm, H Petri (Maastricht), G E von Manteuffel (Marburg), K Irmscher, C Credner, B Baumeister (Mönchengladbach), M Middeke (Munich), V Heinze, H F Benzing, G Mielke (Offenburg), J Zehner, L Weber, D Schmid-Hösl (Passau), H Dobbelstein, W Härtl, W Weber-Guskar (Tutzing), R Ernst (Wiesbaden), F Schardt, W Gross (Würzburg)

This study was supported by a grant from Knoll AG, Ludwigshafen, Federal Republic of Germany.

1 Memorandum from the WHO/ISH. 1986 guidelines for the treatment of mild hypertension. Hypertension 1986;8:957-61.

2 Deutsche Liga zur Bekämpfung des hohen Blutdrucks. Empfehlungen zur Hochdruckbehandlung in der Praxis und zur Behandlung hypertensiver Notfälle. 7th ed. 1986.
Medical Research Council Working Party. MRC trial of treatment of mild hypertension: principal results. Br Med f 1985;291:97-104.

4 The IPPPSH Collaborative Group. Cardiovascular risk and risk factors in randomized trial of treatment based on the beta-blocker oxprenolol: the international prospective primary prevention study in hypertension (IPPPSH). 7 Hypertens 1985;3:379-92.

5 Wilhelmsen L, Berglund G, Elmfeldt D, et al. Beta-blockers versus diuretic in hypertensive men: main results from the HAPPHY trial. $f$ Hyperten 1987;5:561-72.

6 Bühler FR. Age and cardiovascular response adaptation. Determinants of an antihypertensive treatment concept primarily based on beta-blockers and calcium entry blockers. Hypertension 1983;5(suppl III):94-100.

7 Zanchetti A. Role of calcium antagonists in systemic hypertension. Am f Cardiol 1987:59:130-6B.

8 Weber $F$, Anlauf $M$. Direkte und indirekte Vergleichsuntersuchungen zur Meßgenauigkeit des elektronischen Blutdruckmeßgerätes Tonomed. Herz Kreislauf 1982;5:279-83.

9 Breslow NE, Day NE. Statistical methods in cancer research. Vol 1. The analysis of case control studies. Lyons: IARC Scientific Publications, 1980:142-6.

10 Veterans Administration Cooperative Study Group on Antihypertensive Agents. Comparison of propranolol and hydrochlorothiazide for the initial treatment of hypertension. I. Results of short-term titration with emphasis on racial differences in response. $\mathcal{F} A M A$ 1982;248:1996-2003.

11 Freis ED, Materson BJ, Flamenbaum W. Comparison of propranolol and hydrochlorothiazide alone for treatment of hypertension. III. Evaluation of the renin-angiotensin system. Am f Med 1983;74: 1029-41.

12 Frishman WH, Zawada ET, Smith LK, et al. Comparison of hydrochlorothiazide and sustained-release diltiazem for mild-to-moderate systemic hypertension. Am f Cardiol 1987;59:615-23.

13 Berglund G, Andersson O. Low doses of hydrochlorothiazide in hypertension. Antihypertensive and metabolic effects. Eur $\mathcal{F}$ Clin Pharmacol 1976;10 $177-82$

14 Sarachek NS, London RL, Matulewicz TH. Diltiazem and granulomatous hepatitis. Gastroenterology 1985;88:1260-2.

15 Zimmerman HJ, Maddrey WC Toxic and drug-induced hepatitis. In: Schiff L, Schiff ER, eds. Diseases of the liver. 6th ed. Philadelphia: Lippincott, 1987:644.

(Accepted 11 August 1989)
Department of Community Medicine,

Medical School,

Edinburgh EH8 9AG

Vera Carstairs, BSC, honorary

fellow

Russell Morris, BSC, research

officer

Correspondence and requests for reprints to:

Mrs Carstairs.

Br.Med f 1989:299:886-9

\title{
Deprivation: explaining differences in mortality between Scotland and England and Wales
}

\author{
Vera Carstairs, Russell Morris
}

\section{Abstract}

To detect reasons for the difference in mortality between Scotland and England and Wales a measure of deprivation was studied, comprising overcrowding, unemployment of men, low social class, and not having a car. Data for Scotland for 1980-2 showed this measure to be strongly associated with mortality, with gradients being particularly steep in young adults. Deprivation was much more severe in Scotland than in England and Wales. These findings suggest that much excess mortality may be ascribed to more adverse conditions. Standardising the mortality ratios to take account of the relative affluence and deprivation of the two populations led to the differentials observed being radically adjusted, while standardising for social class had little effect. Deprivation measures based on areas overcome many of the limitations associated with social class analysis and also show much greater discrimination between populations.

Measures of deprivation apparently provide a powerful basis for explanation of health differences. Such measures should therefore form part of the 1991 census output to facilitate their use on a consistent basis.

\section{Introduction}

Mortality within the United Kingdom differs, with England having the lowest and Scotland and Northern Ireland vying for the place at the top of the league. Factors that may explain these differences are not easily identified, though selective migration, poorer diet, higher levels of smoking, and the more unfavourable climate may all contribute. We present evidence on the association between mortality and a measure of deprivation in Scotland and England and Wales that suggests that much excess mortality could be associated with the more adverse socioeconomic circumstances experienced in Scotland, deprivation presenting a picture of differences in the populations that is not present in the distribution of social class.

\section{Method}

We calculated deprivation scores for the postcode sectors in Scotland and wards in England and Wales from the 1981 census and mortality for $1980-2$ by age, sex, and category of deprivation for Scotland, which provided the basis for calculating expected deaths in England and Wales. Compared with the observed deaths these provided standardised mortality ratios that took into account differences in the material circumstances of the two populations.

The deprivation measure was based on four census variables: overcrowding, unemployment among men, low social class, and not having a car. These were combined into a single score for each postcode sector in Scotland $(n=$ about $100 \overline{0})$ by means of the $Z$ score technique. The value of each component variable for a sector was standardised to have a mean of zero and standard deviation of 1 by subtracting the mean value for the population (or Scotland) and then dividing by its respective population standard deviation. The four standardised component variables were then added together into one score that had a population mean of zero and standard deviation of 1 . The resulting distribution was split on an arbitrary basis into seven categories ranging from very affluent to very deprived. These categories were designed to maintain the discrimination in the population, with most being allocated to the three middle categories and smalle 